\title{
Multifokale Lymphangioleiomyomatose als Zufallsbefund in der onkologischen Nachsorge einer 80-jährigen Patientin
}

\section{Einleitung}

Die Lymphangioleiomyomatose (LAM) ist eine seltene Systemerkrankung, von der nahezu ausschließlich Frauen betroffen sind, und die aufgrund progredienter pulmonaler Symptomatik üblicherweise prämenopausal manifest wird [Harknett EC, Chang WY, Byrnes S et al. Q J Med 2011; 104: 971-979]. Präsentiert wird ein Fall, in dem die Erkrankung als Zufallsbefund und mit atypischem Befallsmuster bei einer postmenopausalen Patientin hohen Alters erstmals festgestellt wurde.

\section{Fallbeschreibung}

Eine 80-jährige Patientin stellte sich zur regulären Tumornachsorge nach Aderhautmelanom in der dermatologischen Ambulanz vor (Erstdiagnose 5 Jahre zuvor, behandelt mittels Brachytherapie). Bei körperlichem Wohlbefinden fielen laborchemisch ein mit $1,44 \mu \mathrm{g} / \mathrm{l}$ erstmals deutlich erhöhter Tumormarker S $100 \beta$ (Norm $<0,15 \mu \mathrm{g} / \mathrm{l}$, vormals $0,14 \mu \mathrm{g} / \mathrm{l}$ ) sowie eine zunehmend eingeschränkte Nierenfunktion auf (GFR 33 ml/min). Zum Ausschluss von Melanom-Metastasen wurde eine aufgrund der eingeschränkten Nierenfunktion native - Computertomografie (CT) von Thorax und Abdomen ergänzt, welche, trotz der lange zurückliegenden Erstvorstellung der gut bekannten Patientin, die erste vorliegende abdominelle Schnittbildgebung darstellte. Hier zeigten sich multiple pathologisch vergrößerte Lymphknoten mediastinal, retrokrural ( $\triangleright$ Abb. 1a), paravertebral, retroperitoneal und parailiakal ( $\mathbf{A} \mathbf{b} \mathbf{b} . \mathbf{1 b}$ ). Des Weiteren fielen kleinzystische Läsionen beider Lungen ( $\triangleright$ Abb. 2) und ein dilatierter Ductus thoracicus auf ( $\triangleright$ Abb. 3). Weitere Organläsionen fanden sich auch in einer ergänzenden Magnetresonanztomografie (MRT) des Abdomens nicht. Zur Dignitätsklärung der für lymphonodale Melanomfiliae atypisch lokalisierten Lymphknotenvergrößerungen wurde CT-gesteuert eine stanzbioptische Probe aus einem Lymphknoten rechts parailiakal entnommen. Histopathologisch verifizierte sich die Läsion als
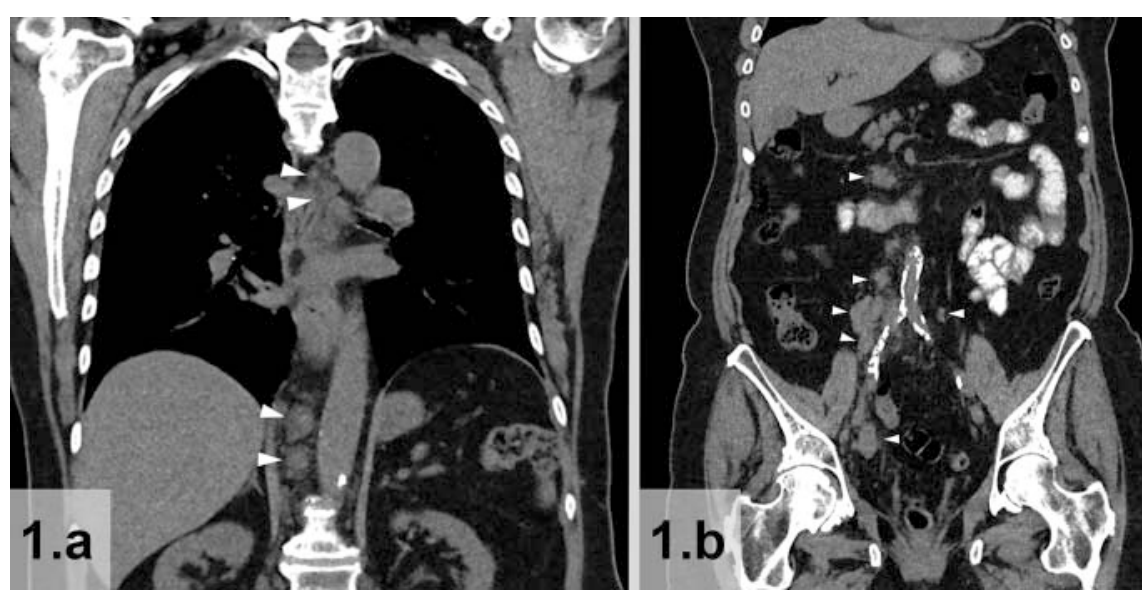

- Abb. 1 CT Thorax und Abdomen (nativ): Lymphadenopathie mediastinal, paraaortal (a, weiße Pfeilspitzen) sowie parailiakal bds. und mesenterial (b, weiße Pfeilspitzen).
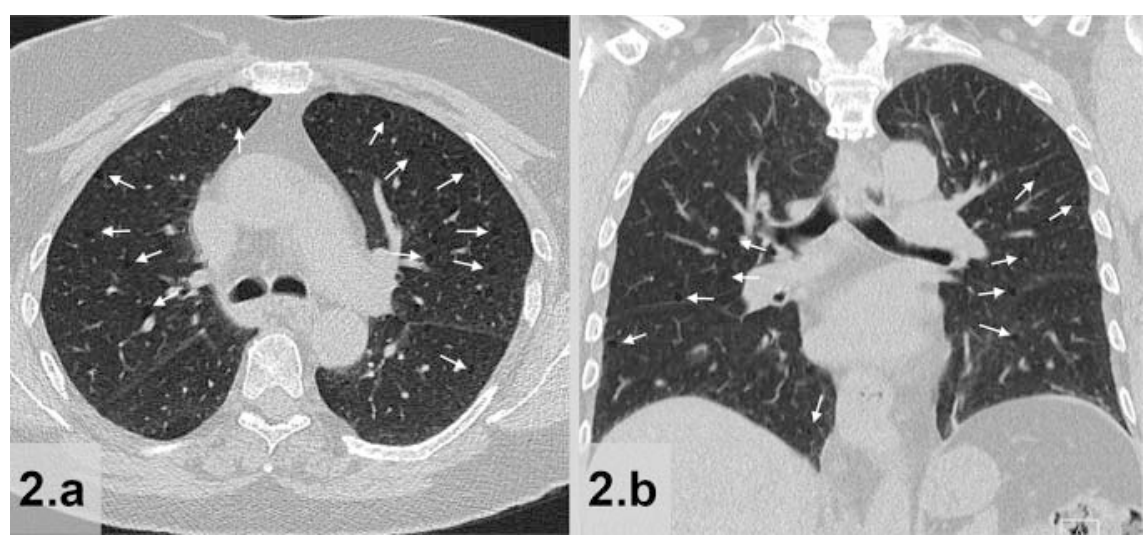

- Abb.2 In den CT-Rekonstruktionen im Lungenfenster zeigen sich zahlreiche kleinzystische Lungenläsionen bipulmonal (weiße Pfeile).

LAM-Befall. Bei auch auf gezielte Nachfrage völliger Beschwerdefreiheit der Patientin wurde seitens der behandelnden Kollegen einvernehmlich auf eine spezifische Therapie verzichtet. Das S $100 \beta$ normalisierte sich im Verlauf, die initiale Erhöhung wurde retrospektiv der eingeschränkten Nierenfunktion zugeschrieben.

\section{Diskussion}

Die LAM ist eine proliferative Multisystemerkrankung der glatten Muskelzellen, die aus einer Mutation im TSC1-/TSC2-Gen resultiert. Sie ist mit einer Prävalenz von ca. 3-8/1000000 Frauen selten und betrifft fast ausschließlich das weibliche
Geschlecht. Patientinnen mit LAM leiden bei Diagnosestellung zumeist unter pulmonalen Symptomen wie Dyspnoe, Hämoptysen, Chylo- oder Pneumothorax resultierend aus progredienten Lungenzysten [Taveira-DaSilva AM, Moss ]. Clin Epidemiol 2015; 7: 249-257]. Seltenere Manifestationen beinhalten mitunter renale Angiomyolipome, Lymphangiome oder eine abdominelle Lymphadenopathie. Studien konnten einen Zusammenhang zwischen abdominellen Manifestationen und Ausprägung der pulmonalen Symptome feststellen, insbesondere korrelierte der Befall abdomineller Lymphknoten signifikant mit der Schwere der Lungenveränderungen [Taylor JR, Ryu ], Colby 


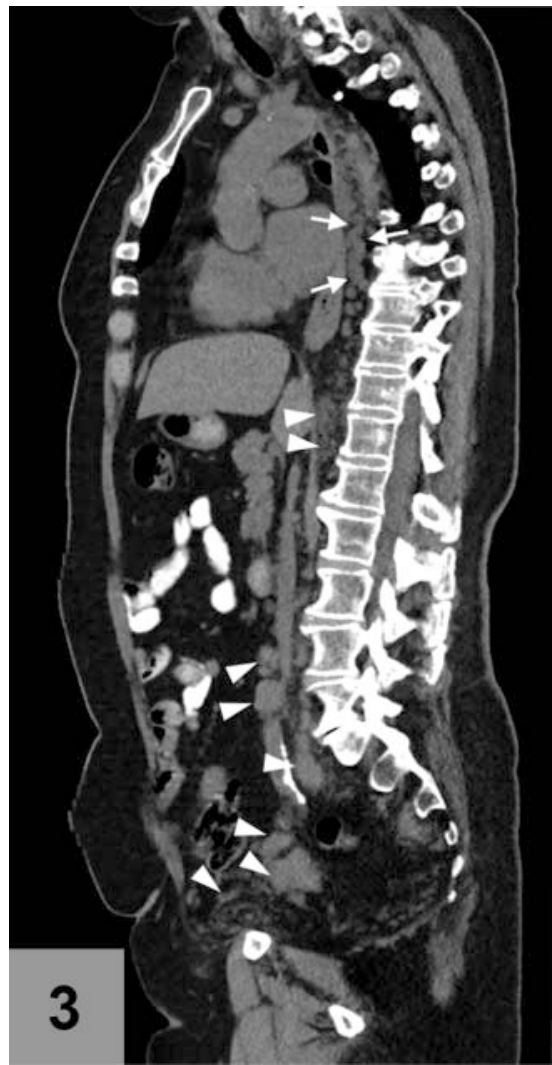

- Abb. 3 In der sagittalen CT-Rekonstruktion kommen der dilatierte Ductus thoracicus (weiße Pfeile) sowie retrokrurale und abdominelle Lymphknotenvergrößerungen (weiße Pfeilspitzen) zur Darstellung.

TV et al. N Engl J Med 1990; 323: $1254-$ 1260; Avila NA et al. Radiology 2000; 216: 147-153]. Dies verdeutlicht das außergewöhnliche Muster des präsentierten Falls, in dem die Patientin trotz der ausgedehnten abdominellen Lymphadenopathie weder pulmonale noch abdominelle Symptome zeigte. Die erst in außergewöhnlich hohem Lebensalter gestellte Erstdiagnose der LAM, deren Manifestationen initial zu diagnostischer Unsicherheit hinsichtlich der Differenzialdiagnose eines metastasierenden Tumorleidens führten, unterstreicht die Eigenart der Kasuistik.

Mögliche Therapieoptionen der LAM sind neben einer Lungentransplantation in erster Linie mTOR-Inhibitoren wie Sirolimus [Taveira-DaSilva AM, Moss ]. Clin Epidemiol 2015; 7: 249-257]. Ein Konsens zur Behandlung solitärer abdomineller Manifestationen existiert insbesondere bei asymptomatischen Patientinnen aufgrund der geringen Fallzahl nicht. Bei abdominellen Symptomen aufgrund der raumfordernden Wirkung von Lymphadenopathie/Lymphangiomen ist als Therapie eine chirurgische Resektion beschrieben [Ding Y, Yan S, Tian Y et al. World J Surg Oncol 2015; 13: 93].

\section{Schlussfolgerung}

Lymphonodale Manifestationen einer LAM können - insbesondere bei Patienten mit einer malignen Grunderkrankung - mit lymphonodalen Metastasen verwechselt werden. Es sollte daher stets eine differenzierte Bildinterpretation erfolgten. Bei diagnostischer Unklarheit kann unter Umständen eine bioptische Sicherung zur Differenzierung erforderlich werden.

\section{KEY POINTS}

- Die LAM ist eine Erkrankung der Frau, die sich in der Regel durch pulmonale Symptome bereits prämenopausal manifestiert.

- Typische radiologische Zeichen sind kleinzystische Lungenläsionen (CT).

- Extrapulmonale Manifestationen der LAM beinhalten unter ande- rem thorakale und abdominelle Lymphknotenvergrößerungen.

- Diese können mit lymphonodalen Metastasen verwechselt werden, sodass unter Umständen eine bioptische Sicherung zur Differenzierung notwendig ist.

Interessenkonflikt

Die Autorinnen/Autoren geben an, dass kein Interessenkonflikt besteht.

\section{Autorinnen/Autoren}

Philipp Josef Kuhl, Philipp Gruschwitz, Simon Veldhoen

Department of Diagnostic and Interventional Radiology, Universitätsklinikum Würzburg, Germany

\section{Korrespondenzadresse}

\author{
Philipp Josef Kuhl \\ Department of Diagnostic and \\ Interventional Radiology, \\ Universitätsklinikum Würzburg \\ Oberdürrbacher Straße 2 \\ 97080 Würzburg \\ Germany \\ Tel.: ++49/931/20134001 \\ kuhl_p@ukw.de
}

\section{Bibliografie}

Fortschr Röntgenstr 2020; 192: 1200-1201

Online-Publikation: 9.6.2020

DOI 10.1055/a-1172-6595

ISSN 1438-9029

(c) 2020. Thieme. All rights reserved.

Georg Thieme Verlag KG, Rüdigerstraße 14, 70469 Stuttgart, Germany

\section{ERRATUM}

Versehentlich wurde die Abbildung 3 in der Druckversion nicht berücksichtigt. 